\title{
2- AND 3-LOOP HEAVY FLAVOR CORRECTIONS TO TRANSVERSITY*
}

\author{
Johannes Blümlein, Sebastian Klein, Beat Tödtli \\ Deutsches Elektronen-Synchrotron DESY, Zeuthen \\ Platanenallee 6, 15738 Zeuthen, Germany
}

(Received October 29, 2009)

We calculate the two- and three-loop massive operator matrix element (OME) contributing to the heavy flavor Wilson coefficients of transversity. We obtain the complete result for the two-loop OME and compute the first thirteen Mellin moments at three-loop order. As a by-product of the calculation, the moments $N=1$ to 13 of the complete two-loop and the $T_{F}$-part of the three-loop transversity anomalous dimension are obtained.

PACS numbers: 12.38.Bx

\section{Framework}

The transversity distribution belongs to the three twist-2 parton distribution functions (PDFs), together with those for unpolarized and polarized deep-inelastic scattering. It is a flavor non-singlet, chiral-odd distribution and can be measured in semi-inclusive deep-inelastic scattering (SIDIS) and via the polarized Drell-Yan process (for a review see Ref. [1]). Different experiments perform transversity measurements at the moment, cf. Refs [2]. Recently, a first phenomenological parameterization has been given for the transversity up- and down-quark distributions in Ref. [3], the moments of which are in qualitative agreement with first lattice calculations [4].

The scattering cross-section for semi-inclusive deeply inelastic charged lepton-nucleon scattering $l N \rightarrow l^{\prime} h+X$ is given by

$$
\begin{aligned}
& \frac{d^{3} \sigma^{\mathrm{SIDIS}}}{d x d y d z}=\frac{4 \pi \alpha_{\mathrm{em}}^{2} s}{Q^{4}} \sum_{a=q, \bar{q}} e_{a}^{2} x\left\{\frac{1}{2}\left[1+(1-y)^{2}\right] F_{a}\left(x, Q^{2}\right) D_{a}\left(z, Q^{2}\right)\right. \\
& \left.-(1-y)\left|\boldsymbol{S}_{\perp}\right|\left|\boldsymbol{S}_{h \perp}\right| \cos \left(\phi_{S}+\phi_{S_{h}}\right) \Delta_{\mathrm{T}} F_{a}\left(x, Q^{2}\right) \Delta_{\mathrm{T}} D_{a}\left(z, Q^{2}\right)\right\}
\end{aligned}
$$

* Presented at the XXXIII International Conference of Theoretical Physics, "Matter to the Deepest", Ustroń, Poland, September 11-16, 2009. 


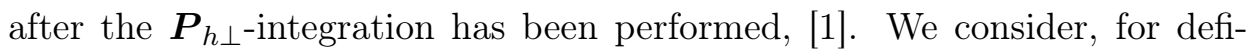
niteness, only scattering cross-sections free of $\boldsymbol{k}_{\perp}$-effects to refer to twist-2 quantities. $x$ and $y$ denote the Bjorken variables, $z$ the fragmentation variable, $q^{2}=-Q^{2}$ the space-like 4-momentum transfer, $\alpha_{\mathrm{em}}$ the fine structure constant, $e_{a}$ the quark charge, and $s$ the c.m.s. energy squared. $\boldsymbol{S}_{\perp}$ and $\boldsymbol{S}_{h \perp}$ are the transverse spin vectors of the incoming nucleon $N$ and the measured hadron $h . F_{a}\left(z, Q^{2}\right), \Delta_{\mathrm{T}} F_{a}\left(z, Q^{2}\right)$ and $D_{a}\left(z, Q^{2}\right), \Delta_{\mathrm{T}} D_{a}\left(z, Q^{2}\right)$ denote the unpolarized and transversity structure- and fragmentation functions, respectively. The angles $\phi_{S, S_{h}}$ are measured in the plane transverse to the $\gamma^{*} N$ axis between the $x$-axis and the respective vector. In process (1) the spin of the transversely polarized hadron $h$ has to be measured.

The transversity distribution may also be measured in the transversely polarized Drell-Yan processes. In Mellin space the scattering cross-section is given by [5]

$$
\frac{d \Delta_{\mathrm{T}} \sigma^{\mathrm{DY}}}{d \phi}=\frac{\alpha_{\mathrm{em}}^{2}}{9 s} \cos (2 \phi) \Delta_{\mathrm{T}} H\left(N, M^{2}\right) \Delta_{\mathrm{T}} C_{q}^{\mathrm{DY}}\left(N, M^{2}\right),
$$

where $N$ denotes the Mellin variable and $\phi$ is the azimuthal angle of one of the final state leptons $l^{ \pm}$relative to the axis defined by the transverse polarizations.

$$
\Delta_{\mathrm{T}} H\left(N, Q^{2}\right)=\sum_{q} e_{q}^{2}\left[\Delta_{\mathrm{T}} q_{1}\left(N, Q^{2}\right) \Delta_{\mathrm{T}} \bar{q}_{2}\left(N, Q^{2}\right)+\Delta_{\mathrm{T}} \bar{q}_{1}\left(N, Q^{2}\right) \Delta_{\mathrm{T}} q_{2}\left(N, Q^{2}\right)\right]
$$

is a combination of transversity parton distributions for the incoming light (anti-)quarks, and $\Delta_{\mathrm{T}} C_{q}^{\mathrm{DY}}\left(N, M^{2}\right)$ denotes the Wilson coefficient of the Drell-Yan process, with $M^{2}$ the invariant mass of the produced lepton pair.

Like in the case of unpolarized and polarized deep-inelastic processes transversity receives heavy flavor corrections in higher orders in QCD. These are given by the corresponding heavy flavor Wilson coefficients. As for other non-singlet quantities [6,7], these corrections start at $O\left(a_{\mathrm{s}}^{2}\right)$, with $a_{\mathrm{s}}=$ $\alpha_{\mathrm{s}} /(4 \pi)$. In SIDIS one can tag $Q \bar{Q}$-production in the same way as in the deep-inelastic process, [8]. A measurement is possible in high luminosity experiments. In the Drell-Yan process, on the other hand, heavy flavor contributions emerge inclusively since there the final-state $l^{+} l^{-}$-pairs are measured in the first place. The calculation of the heavy quark Wilson coefficients for $Q^{2} \gg m^{2}$ proceeds in the same way as in unpolarized and polarized deep-inelastic scattering $[6,7,9,10]$.

The complete Wilson coefficients for transversity can be decomposed into a light- and a heavy quark contribution

$$
C_{q}^{\mathrm{TR}}\left(x, \frac{Q^{2}}{\mu^{2}}, \frac{m^{2}}{\mu^{2}}\right)=C_{q}^{\mathrm{TR}, \text { light }}\left(x, \frac{Q^{2}}{\mu^{2}}\right)+H_{q}^{\mathrm{TR}}\left(x, \frac{Q^{2}}{\mu^{2}}, \frac{m^{2}}{\mu^{2}}\right) .
$$


As shown in [6], the heavy quark Wilson coefficient for hard processes factorizes into the light quark Wilson coefficients and the massive operator matrix element $A_{q q, Q}^{\mathrm{TR}}$ at large enough scales $Q^{2} \gg m^{2}$. We apply this to the heavy flavor Wilson coefficient for transversity ${ }^{1}$

$$
\begin{aligned}
H_{q}^{\mathrm{TR}}\left(x, \frac{Q^{2}}{\mu^{2}}, \frac{m^{2}}{\mu^{2}}\right)= & C_{q}^{\mathrm{TR}, \operatorname{light}}\left(x, \frac{Q^{2}}{\mu^{2}}\right) \otimes A_{q q, Q}^{\mathrm{TR}}\left(x, \frac{m^{2}}{\mu^{2}}\right) \\
= & a_{\mathrm{s}}^{2}\left[\Delta_{\mathrm{T}} A_{q q, Q}^{(2), \mathrm{NS}, \mathrm{TR}}+\Delta_{\mathrm{T}} \hat{C}_{q}^{(2)}\left(N_{f}\right)\right] \\
& +a_{\mathrm{s}}^{3}\left[\Delta_{\mathrm{T}} A_{q q, Q}^{(3), \mathrm{NS}, \mathrm{TR}}\left(N_{f}+1\right)+\hat{C}_{q}^{(3)}\left(N_{f}\right)\right. \\
& \left.+\Delta_{\mathrm{T}} A_{q q, Q}^{(2), \mathrm{NS}, \mathrm{TR}} \otimes \Delta_{\mathrm{T}} C_{q}^{(1)}\right] .
\end{aligned}
$$

The aim of this article is to present a computation of the renormalized two- and three-loop heavy-flavor operator matrix elements contributing to transversity. Details of the calculation are given in Ref. [11]. The operator matrix element $\left\langle q\left|O^{\mathrm{NS}, \mathrm{TR}}\right| q\right\rangle$ is given by a two-point Green's function containing a closed loop of a heavy quark $Q$ and external massless quarks $q$. The local operator is given by [12]

$$
O_{F, a ; \mu \mu_{1} \ldots \mu_{n}}^{\mathrm{NS}}=i^{n} \boldsymbol{S}\left[\bar{\psi} \gamma_{5} \sigma_{\mu \mu_{1}} D_{\mu_{2}} \ldots D_{\mu_{n}} \frac{\lambda_{a}}{2} \psi\right]-\text { trace terms . }
$$

Here, $\sigma_{\mu \nu}=(i / 2)\left[\gamma_{\mu} \gamma_{\nu}-\gamma_{\nu} \gamma_{\mu}\right], \boldsymbol{S}$ denotes symmetrization of the Lorentz indices and $D_{\mu}$ is the covariant derivative. The Green's function in $D=4+\varepsilon$ dimensions obeys the following vector decomposition

$$
\begin{aligned}
\hat{G}_{\mu, q, Q}^{i j, \mathrm{TR}, \mathrm{NS}}= & J_{N}\left\langle q\left|O_{F, a ; \mu \mu_{1} \ldots \mu_{n}}^{\mathrm{NS}, \mathrm{TR}}\right| q\right\rangle=\delta_{i j}\left\{\Delta_{\rho} \sigma^{\mu \rho} \hat{\hat{A}}_{q q, Q}^{\mathrm{TR}, \mathrm{NS}}\left(\frac{\hat{m}^{2}}{\mu^{2}}, \varepsilon, N\right)\right. \\
& \left.+c_{1} \Delta^{\mu}+c_{2} p^{\mu}+c_{3} \gamma^{\mu} \not p+c_{4} \Delta \not p \Delta^{\mu}+c_{5} \Delta \not p p^{\mu}\right\}(\Delta \cdot p)^{N-1}
\end{aligned}
$$

contracting the OME with a source term $J_{N}=\Delta^{\mu_{1}} \ldots \Delta^{\mu_{N}}$, with $\Delta^{2}=0$ and $p$ the parton momentum. It determines the un-renormalized massive OME

$$
\begin{aligned}
\hat{\hat{A}}_{q q, Q}^{\mathrm{TR}, \mathrm{NS}}\left(\frac{\hat{m}^{2}}{\mu^{2}}, \varepsilon, N\right)= & \frac{-i \delta^{i j}}{4 N_{c}(\Delta \cdot p)^{N+1}(D-2)}\left\{\operatorname{Tr}\left[\Delta \not p p^{\mu} \hat{G}_{\mu, q, Q}^{i j, \mathrm{TR}, \mathrm{NS}}\right]\right. \\
& \left.-\Delta \cdot p \operatorname{Tr}\left[p^{\mu} \hat{G}_{\mu, q, Q}^{i j, \mathrm{TR}, \mathrm{NS}}\right]+i \Delta \cdot p \operatorname{Tr}\left[p^{\mu} \hat{G}_{\mu, q, Q}^{i j, \mathrm{TR}, \mathrm{NS}}\right]\right\}
\end{aligned}
$$

${ }^{1}$ We use the notation $\hat{f}\left(N_{f}\right)=f\left(N_{f}+1\right)-f\left(N_{f}\right)$. 
A total of 129 diagrams contribute, which were generated using QGRAF [13]. These were projected onto $\hat{A}_{q q, Q}^{\mathrm{TR}, \mathrm{NS}}, c f$. [9], using codes written in FORM [14]. After tensor reduction, the loop integrals are of the tadpole-type, since the single external quark is on-shell and massless. The integrals were then evaluated using MATAD [15]. The renormalization of the OMEs is described in Ref. [9].

After mass- and charge renormalization one obtains the massive OMEs in the on-mass-shell scheme, $c f$. [9],

$$
\begin{aligned}
\Delta_{\mathrm{T}} A_{q q, Q}^{(2), \mathrm{NS}, \overline{\mathrm{MS}}}= & \frac{\beta_{0, Q} \gamma_{q q}^{(0), \mathrm{TR}}}{4} \ln ^{2}\left(\frac{m^{2}}{\mu^{2}}\right)+\frac{\hat{\gamma}_{q q}^{(1), \mathrm{TR}}}{2} \ln \left(\frac{m^{2}}{\mu^{2}}\right)+a_{q q, Q}^{(2), \mathrm{TR}}-\frac{\beta_{0, Q} \gamma_{q q}^{(0), \mathrm{TR}}}{4} \zeta_{2}, \\
\Delta_{\mathrm{T}} A_{q q, Q}^{(3), \mathrm{NS}, \overline{\mathrm{MS}}}= & -\frac{\gamma_{q q}^{(0), \mathrm{TR}} \beta_{0, Q}}{6}\left(\beta_{0}+2 \beta_{0, Q}\right) \ln ^{3}\left(\frac{m^{2}}{\mu^{2}}\right)+\frac{1}{4}\left\{2 \gamma_{q q}^{(1), \mathrm{TR}} \beta_{0, Q}\right. \\
& \left.-2 \hat{\gamma}_{q q}^{(1), \mathrm{TR}}\left(\beta_{0}+\beta_{0, Q}\right)+\beta_{1, Q} \gamma_{q q}^{(0), \mathrm{TR}}\right\} \ln ^{2}\left(\frac{m^{2}}{\mu^{2}}\right)+\frac{1}{2}\left\{\hat{\gamma}_{q q}^{(2), \mathrm{TR}}\right. \\
& \left.-\left(4 a_{q q, Q}^{(2), \mathrm{TR}}-\zeta_{2} \beta_{0, Q} \gamma_{q q}^{(0), \mathrm{TR}}\right)\left(\beta_{0}+\beta_{0, Q}\right)+\gamma_{q q}^{(0), \mathrm{TR}} \beta_{1, Q}^{(1)}\right\} \ln \left(\frac{m^{2}}{\mu^{2}}\right) \\
& +4 \bar{a}_{q q, Q}^{(2), \mathrm{TR}}\left(\beta_{0}+\beta_{0, Q}\right)-\gamma_{q q}^{(0)} \beta_{1, Q}^{(2)}-\frac{\gamma_{q q}^{(0), \mathrm{TR}} \beta_{0} \beta_{0, Q} \zeta_{3}}{6}-\frac{\gamma_{q q}^{(1), \mathrm{TR}} \beta_{0, Q} \zeta_{2}}{4} \\
& +2 \delta m_{1}^{(1)} \beta_{0, Q} \gamma_{q q}^{(0), \mathrm{TR}}+\delta m_{1}^{(0)} \hat{\gamma}_{q q}^{(1), \mathrm{TR}}+2 \delta m_{1}^{(-1)} a_{q q, Q}^{(2), \mathrm{TR}}+a_{q q, Q}^{(3), \mathrm{TR}}
\end{aligned}
$$

at 2- and 3-loops. Here, $\zeta_{k}$ denotes the Riemann $\zeta$-function and $\gamma_{q q}^{(l), T R}$ are the transversity anomalous dimensions for $l=0,1,2$ in LO [16], NLO [5, 17], and NNLO [18]. For the other quantities we refer to [9]. The new terms being calculated are $a_{q q, Q}^{(2), \mathrm{TR}}(N), \bar{a}_{q q, Q}^{(2), \mathrm{TR}}(N)$ and $a_{q q, Q}^{(3), \mathrm{TR}}(N)$, and for the higher values of $N, \hat{\gamma}_{q q}^{(2), \mathrm{TR}}(N)$.

\section{Results}

\subsection{Massive operator matrix elements}

At $O\left(a_{\mathrm{s}}^{2}\right)$ the massive operator matrix elements for transversity $\Delta_{\mathrm{T}} A_{q q, Q}^{(2), \mathrm{NS}, \overline{\mathrm{MS}}}$ are obtained for general values of $N, c f$. Eq. (8). The un-renormalized OME is computed to $O(\varepsilon)$ to also extract the function $\bar{a}_{q q, Q}^{(2), \mathrm{TR}}(N)$. The new terms at 2-loops are $a_{q q, Q}^{(2), \mathrm{TR}}$ and $\bar{a}_{q q, Q}^{(2), \mathrm{TR}}, c f$. Eqs $(8),(9)$ :

$$
a_{q q, Q}^{\mathrm{TR},(2)}(N)=C_{F} T_{F}\left\{-\frac{8}{3} S_{3}+\frac{40}{9} S_{2}-\left[\frac{224}{27}+\frac{8}{3} \zeta_{2}\right] S_{1}+2 \zeta_{2}+\frac{\left(24+73 N+73 N^{2}\right)}{18 N(1+N)}\right\}
$$




$$
\begin{aligned}
\bar{a}_{q q, Q}^{\mathrm{TR},(2)}(N)= & C_{F} T_{F}\left\{-\left[\frac{656}{81}+\frac{20}{9} \zeta_{2}+\frac{8}{9} \zeta_{3}\right] S_{1}+\left[\frac{112}{27}+\frac{4}{3} \zeta_{2}\right] S_{2}-\frac{20}{9} S_{3}\right. \\
& \left.+\frac{4}{3} S_{4}+\frac{1}{6} \zeta_{2}+\frac{2}{3} \zeta_{3}+\frac{\left(-144-48 N+757 N^{2}+1034 N^{3}+517 N^{4}\right)}{216 N^{2}(1+N)^{2}}\right\},
\end{aligned}
$$

with $S_{k} \equiv S_{k}(N)$ denoting the single harmonic sums.

At $O\left(a_{\mathrm{s}}^{3}\right)$ the moments $N=1$ to 13 were computed for the massive $\mathrm{OME}$, as $e . g$.

$$
\begin{aligned}
& \Delta_{\mathrm{T}} A_{q q, Q}^{(3), \mathrm{NS}, \overline{\mathrm{MS}}}(13)=C_{F} T_{F}\left\{\left[\frac{1751446}{110565} C_{A}-\frac{7005784}{1216215} T_{F}\left(N_{f}+2\right)\right] \ln ^{3}\left(\frac{m^{2}}{\mu^{2}}\right)\right. \\
& -\left[\frac{20032048197492631}{2193567563187000} C_{F}+\frac{137401473299}{8027019000} C_{A}+\frac{93611152819}{3652293645} T_{F}\right] \ln ^{2}\left(\frac{m^{2}}{\mu^{2}}\right) \\
& +\left[\left(\frac{1705832327329042449983}{263491335690022440000}+\frac{7005784}{45045} \zeta_{3}\right) C_{F}+\left(\frac{3385454488248191237}{65807026895610000}\right.\right. \\
& \left.-\left(\frac{7005784}{45045} \zeta_{3}\right) C_{A}-\frac{458114791076413771}{6580702689561000} N_{f} T_{F}-\frac{217179304}{3648645} T_{F}\right] \ln \left(\frac{m^{2}}{\mu^{2}}\right) \\
& +\left(\frac{3502892}{135135} B_{4}-\frac{3502892}{15015} \zeta_{4}+\frac{4061479439}{12162150} \zeta_{3}-\frac{3486896974743882556775647}{12935029206601101600000}\right) C_{A} \\
& -\left(\frac{279922752632160355860697}{3557133031815302940000}-\frac{56046272}{1216215} \zeta_{3}\right) T_{F} N_{f} \\
& \left.+\left(\frac{291526550302760070155303}{7114266063630605880000}-\frac{14011568}{173745} \zeta_{3}\right) T_{F}\right\},
\end{aligned}
$$

where

$$
B_{4}=-4 \zeta_{2} \ln ^{2}(2)+\frac{2}{3} \ln ^{4}(2)-\frac{13}{2} \zeta_{4}+16 \mathrm{Li}_{4}\left(\frac{1}{2}\right)
$$

Like for the massive OMEs in case of unpolarized deep-inelastic scattering the structure of $\Delta_{\mathrm{T}} A_{q q, Q}^{(3), \mathrm{NS}, \overline{\mathrm{MS}}}(N)$ is widely known for general values of $N$, except for the finite part $a_{q q, Q}^{(3), N S, T R}$ and the 3-loop anomalous dimension $\hat{\gamma}_{q q}^{(2), \mathrm{TR}}(N)$. One notices the cancellation of all $\zeta_{2}$ terms in $\Delta_{\mathrm{T}} A_{q q, Q}^{(3), \mathrm{NS}, \overline{\mathrm{MS}}}(N)$ after renormalization.

\subsection{Anomalous dimensions}

The transversity anomalous dimension is given by

$$
\gamma_{q q}^{\mathrm{TR}}\left(N, a_{\mathrm{s}}\right)=\sum_{i=1}^{\infty} a_{\mathrm{s}}^{i} \gamma_{q q}^{(i), \mathrm{TR}}(N)
$$


From Eq. (9) one may determine the complete 2-loop anomalous dimension $[5,17]$ and the $T_{F}$-part of the 3-loop anomalous dimension [18]. We agree with the results given in $[5,17]$ and confirm the $T_{F}$-contributions for the moments $N=1$ to 8 given in Ref. [18]. Furthermore, we newly obtain $\hat{\gamma}_{q q}^{(2), \mathrm{TR}}=\gamma_{q q}^{(2), \mathrm{TR}}\left(N_{f}+1\right)-\gamma_{q q}^{(2), \mathrm{TR}}\left(N_{f}\right)$ for $N=9$ to 13 , as e.g.

$$
\begin{aligned}
& \hat{\gamma}_{q q}^{(2), \mathrm{TR}}(N=13)=-C_{F} T_{F}\left[\frac{36713319015407141570017}{131745667845011220000} C_{F}-\frac{14011568}{45045}\right. \\
& \left.\times\left(C_{F}-C_{A}\right) \zeta_{3}+\frac{66409807459266571}{3290351344780500} T_{F}\left(1+2 N_{f}\right)+\frac{6571493644375020121}{65807026895610000} C_{A}\right] .
\end{aligned}
$$

\subsection{A remark on the Soffer bound}

If the Soffer inequality [19]

$$
\left|\Delta_{\mathrm{T}} f\left(x, Q^{2}\right)\right| \leq \frac{1}{2}\left[f\left(x, Q^{2}\right)+\Delta f\left(x, Q^{2}\right)\right]
$$

holds for the non-perturbative PDF in Eq. (15) one may check its generalization from $f_{i} \rightarrow F_{i}$ for the corresponding structure functions. This includes the non-singlet evolution operator (Eq. (6), Ref. [20]) and the heavy flavor Wilson coefficient. At perturbative scales, it holds for the evolution operator [11], generalizing a NLO result from [5] to the moments $N=1$ to 13 at NNLO. For the heavy quark Wilson coefficient in SIDIS we only know the massive OME so far. As shown in Ref. [11], a final conclusion can only be drawn knowing the yet undetermined massless Wilson coefficients. The difference $\left[A_{q q, Q}^{\mathrm{V}}-A_{q q, Q}^{\mathrm{TR}}\right](x)$ of the massive OMEs, shows a sign change to negative values for $Q^{2} / m^{2}$ in the physical range. For large scales $Q^{2} / m^{2} \gg 1$ positive values are obtained.

\section{REFERENCES}

[1] V. Barone, A. Drago, P.G. Ratcliffe, Phys. Rep. 359, 1 (2002) [hep-ph/0104283].

[2] A. Airapetian et al. [HERMES Collaboration], Phys. Rev. Lett. 94, 012002 (2005) [hep-ex/0408013]; A. Airapetian et al. [HERMES Collaboration], J. High Energy Phys. 0806, 017 (2008) [arXiv:0803.2367 [hep-ex]]; M. Alekseev et al. [COMPASS Collaboration], Phys. Lett. B673, 127 (2009) [arXiv:0802.2160 [hep-ex]]; V.Y. Alexakhin et al. [COMPASS Collaboration], Phys. Rev. Lett. 94, 202002 (2005) [hep-ex/0503002]; COMPASS Collaboration, private communication; M.F. Lutz, et al., [The PANDA Collaboration], arXiv:0903.3905 [hep-ex]; A. Afanasev et al., hep-ph/0703288. 
[3] M. Anselmino et al., arXiv:0807.0173 [hep-ph].

[4] S. Aoki, M. Doui, T. Hatsuda, Y. Kuramashi, Phys. Rev. D56, 433 (1997) [hep-lat/9608115]; M. Göckeler et al., Nucl. Phys. Proc. Suppl. 53, 315 (1997) [hep-lat/9609039]; A. Ali Khan et al., Nucl. Phys. Proc. Suppl. 140, 408 (2005) [hep-lat/0409161]; D. Dolgov et al. [LHPC Collaboration and TXL Collaboration], Phys. Rev. D66, 034506 (2002) [hep-lat/0201021]; M. Diehl et al. [QCDSF Collaboration and UKQCD Collaboration], hep-ph/0511032; M. Göckeler et al. [QCDSF Collaboration and UKQCD Collaboration], Phys. Rev. Lett. 98, 222001 (2007) [hep-lat/0612032]; D. Renner, private communication.

[5] W. Vogelsang, Phys. Rev. D57, 1886 (1998) [hep-ph/9706511].

[6] M. Buza, Y. Matiounine, J. Smith, R. Migneron, W.L. van Neerven, Nucl. Phys. B472, 611 (1996) [hep-ph/9601302]; M. Buza, Y. Matiounine,

J. Smith, W.L. van Neerven, Nucl. Phys. B485, 420 (1997) [hep-ph/9608342].

[7] I. Bierenbaum, J. Blümlein, S. Klein, Nucl. Phys. B780, 40 (2007) [hep-ph/0703285]; I. Bierenbaum, J. Blümlein, S. Klein, C. Schneider, Nucl. Phys. B803, 1 (2008) [arXiv:0803.0273 [hep-ph]].

[8] S. Alekhin, J. Blümlein, S. Klein, S. Moch, arXiv:0908.2766 [hep-ph].

[9] I. Bierenbaum, J. Blümlein, S. Klein, Nucl. Phys. B820, 417 (2009) [arXiv:0904.3563 [hep-ph]]; arXiv:0907.2615 [hep-ph].

[10] M. Buza, Y. Matiounine, J. Smith, W.L. van Neerven, Eur. Phys. J. C1, 301 (1998) [hep-ph/9612398]; J. Blümlein, A. De Freitas, W.L. van Neerven, S. Klein, Nucl. Phys. B755, 272 (2006) [hep-ph/0608024]; I. Bierenbaum, J. Blümlein, S. Klein, Phys. Lett. B672, 401 (2009) [arXiv:0901.0669 [hep-ph]]; arXiv:0706.2738 [hep-ph].

[11] J. Blümlein, S. Klein, B. Tödtli, arXiv:0909.1547 [hep-ph].

[12] B. Geyer, D. Robaschik, E. Wieczorek, Fortsch. Phys. 27, 75 (1979).

[13] P. Nogueira, J. Comput. Phys. 105, 279 (1993).

[14] J.A.M. Vermaseren, math-ph/0010025.

[15] M. Steinhauser, Comput. Phys. Commun. 134, 335 (2001) [hep-ph/0009029].

[16] F. Baldracchini, N.S. Craigie, V. Roberto, M. Socolovsky, Fortsch. Phys. 30, 505 (1981); M.A. Shifman, M.I. Vysotsky, Nucl. Phys. B186, 475 (1981); A.P. Bukhvostov, G.V. Frolov, L.N. Lipatov, E.A. Kuraev, Nucl. Phys. B258, 601 (1985); J. Blümlein, Eur. Phys. J. C20, 683 (2001) [hep-ph/0104099]; A. Mukherjee, D. Chakrabarti, Phys. Lett. B506, 283 (2001) [hep-ph/0102003].

[17] A. Hayashigaki, Y. Kanazawa, Y. Koike, Phys. Rev. D56, 7350 (1997) [hep-ph/9707208]; S. Kumano, M. Miyama, Phys. Rev. D56, 2504 (1997) [hep-ph/9706420].

[18] J.A. Gracey, Nucl. Phys. B662, 247 (2003) [hep-ph/0304113]; Nucl. Phys. B667, 242 (2003) [hep-ph/0306163]; J. High Energy Phys. 0610, 040 (2006) [hep-ph/0609231]; Phys. Lett. B643, 374 (2006) [hep-ph/0611071]. 
[19] J. Soffer, Phys. Rev. Lett. 74, 1292 (1995) [hep-ph/9409254].

[20] J. Blümlein, H. Böttcher, A. Guffanti, Nucl. Phys. B774, 182 (2007) [hep-ph/0607200].

[21] Slides: http://prac.us.edu.pl/ us2009/talks/Toedtli.pdf 